\title{
The Saudi Data \& Artificial Intelligence Authority (SDAIA) Vision: Leading the Kingdom's Journey toward Global Leadership
}

\author{
Ziad A. Memish ${ }^{1,2, *}$ (D), Majid M. Altuwaijri ${ }^{3}$, Abdulgader H. Almoeen ${ }^{3}$, Sarah M. Enani ${ }^{3}$ \\ ${ }^{1}$ Research and Innovative Centre, King Saud Medical City, Ministry of Health \& Faculty of Medicine, Alfaisal University, Riyadh, Kingdom of Saudi Arabia \\ ${ }^{2}$ Hubert Department of Global Health, Rollins School of Public Health, Emory University, Atlanta, GA, USA \\ ${ }^{3}$ National Center for Artificial Intelligence (NCAI), Saudi Data and Artificial Intelligence Authority (SDAIA), Riyadh, Kingdom of Saudi Arabia
}

\section{ARTICLE INFO}

\section{Article History}

Received 04 April 2021

Accepted 05 April 2021

Keywords

Artificial intelligence

Saudi Arabia

global

\begin{abstract}
The emerging field of data and artificial intelligence (AI) and its noticeable expansion has motivated nations to transition from traditional economic models to learning economies using these technologies. In April 2016, the Crown Prince Mohammed bin Salman Al Saud introduced Saudi Arabia's Vision 2030 that identifies the long-term goals and expectations of the Kingdom of Saudi Arabia (KSA). The Saudi Data and Artificial Intelligence Authority (SDAIA) was established in August 2019 by a Royal Decree to facilitate the transition and help achieve Vision 2030's goals and reach KSA's fullest potentials. SDAIA's core mandate is to support and drive the data and AI agenda within the Kingdom, and its vision is to position KSA as a global leader in the elite league of data-driven economies. To properly fulfill its mandate, SDAIA announced in October 2020 the National Strategy for Data and AI (NSDAI) with an ambitious vision put in place "Where the best of Data and AI is made reality".
\end{abstract}

(C) 2021 The Authors. Published by Atlantis Press International B.V. This is an open access article distributed under the CC BY-NC 4.0 license (http://creativecommons.org/licenses/by-nc/4.0/).

\section{INTRODUCTION}

The emerging field of data and Artificial Intelligence (AI) and its noticeable expansion has motivated nations to transition from traditional economic models to learning economies through the use of these technologies. With the expansion of data volume and exponential growth of computing powers, various applications in the field of data and AI have been introduced into the real world, where data in particular has become a critical resource for countries, some calling it "the new oil" [1].

The health sector is one of the main benefiters of advanced technologies since healthcare has been growing increasingly complex to physicians, patients, and governments. For instance, the rising costs of healthcare services, especially in high- and middle-income countries, and rising expectations of healthcare quality necessitates a transformational shift in this field. To ensure that healthcare reaches its full potential, it must be modernized by incorporating information technology, health informatics, and AI, or in other words, data and AI technology. Ever since information technology was introduced, it has changed the practice of healthcare providers as well as the way healthcare systems operate by easing and facilitating the processing, storage, and exchange of health information. Furthermore, health informatics allows for the collection and analyzation of health data for the purpose of improving health and healthcare. Artificial intelligence, on the other hand, refers to the use of complex algorithms to perform specific tasks in an

"Corresponding author. Email: zmemish@yahoo.com automated manner. When it comes to health applications, the possibilities of AI are seemingly limitless, ranging from wearable devices and mobile apps to complex diagnostic devices. The ability of AI to process and interpret massive datasets in very short periods of time results in data-driven decisions that is capable of assisting physicians, managing patients' data, and suggesting solutions to complex medical problems [2,3]. Moreover, data and AI technology will pave the way public health operates as it will elevate disease prevention and treatment measures, resulting in significant benefits in terms of health as well as a reduction in the burden of diseases (reduced costs).

In April 2016, the Crown Prince Mohammed bin Salman Al Saud introduced Saudi Arabia's Vision 2030 that identifies the long-term goals and expectations of the Kingdom of Saudi Arabia (KSA). The Saudi Data and Artificial Intelligence Authority (SDAIA) was established in August 2019 by a Royal Decree to facilitate the transition and help achieve Vision 2030's goals and reach KSA's fullest potentials, where SDAIA's governance structure is shown in Figure 1. SDAIA's core mandate is to support and drive the data and AI agenda within the Kingdom, and its vision is to position KSA as a global leader in the elite league of data-driven economies. To properly fulfill its mandate, SDAIA announced in October 2020 the National Strategy for Data and AI (NSDAI) with an ambitious vision put in place "Where the best of Data and AI is made reality". The aim is to achieve the ambitious vision through a multi-phased approach, focusing on addressing national urgencies by 2025 , building foundations for competitive advantage in key niche areas by 2030, and becoming one of the leading economies utilizing and exporting data and AI after 2030. The three 
core entities within SDAIA are the National Data Management Office (NDMO), the National Information Center (NIC), and the National Center for Artificial Intelligence (NCAI) that help deliver the promise of a data-driven and AI-supported government and economy.

In Saudi Arabia, the Government has been significantly increasing its investments in healthcare to accommodate growing demands of its population. The Ministry of Health continues to provide free health services, accounting for $60 \%$ of all health services in the Kingdom, with the remainder provided by others, including the private sector. With over 484 hospitals in Saudi Arabia providing approximately 2.25 hospital beds for every 1000 people and overall medical advancement, the average life expectancy has increased from 66 to 74 years in the last three decades [4,5]. Nonetheless, the healthcare system continues to face major challenges as the population of Saudi Arabia is growing at an annual rate of $2.43 \%$ and as the elderly population is rapidly increasing with a growth rate of $3.2 \%$, where it is expected that $25 \%$ of the Saudi population will be aged 60 or older by the end of 2050 [5,6]. In addition, the rise of global health challenges, as well as the local increase of non-communicable and chronic diseases such as diabetes and cardiovascular diseases, urges the government to prioritize public health measures.

Saudi Vision 2030 has added new challenges, as well as opportunities, for the healthcare sector to grow and thrive. SDAIA has identified the healthcare sector as a national priority due to the importance of data and AI technology in meeting the sector's growing demands. Moreover, data and AI could be used to target three of Vision 2030's strategic objectives shown in Figure 2 - ease the access of healthcare services, improve value of healthcare services, and strengthen prevention against health threats - to help achieve the overarching goal of increasing life expectancy from 74 to 80 years and improving the quality of life.

There is a significant role and numerous opportunities for data and AI to be used in the health sector, and these opportunities will be lead, driven, and supported by SDAIA in aims of advancing

\section{$\angle$ New vision led to the development and approval of a new national data management governance model}

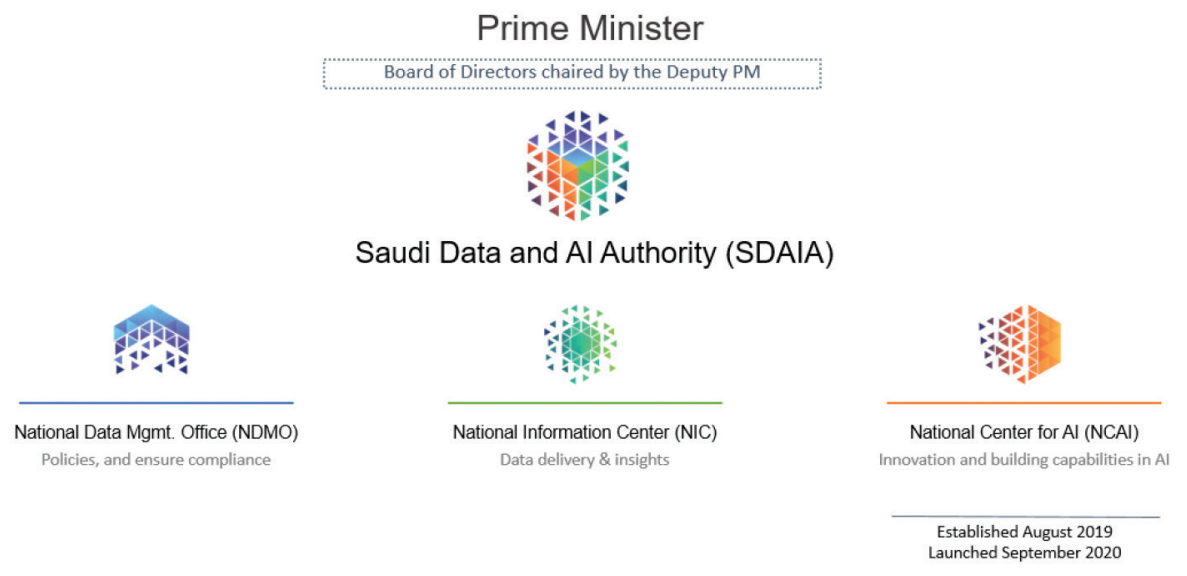

Figure 1 SDAIA’s governance structure.

\section{Within Vision 2030, 3 strategic objectives are directly linked to the Healthcare sector}

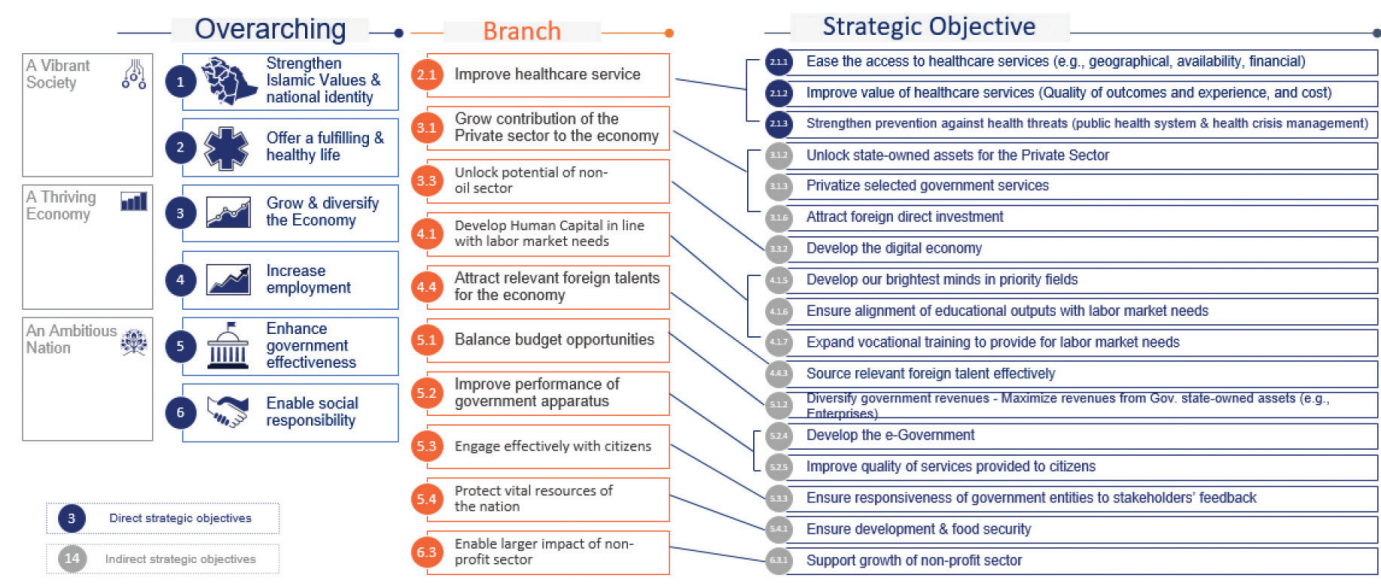

Figure 2 Vision 2030's strategic objectives. 


\section{Data and Al have the potential to provide several benefits relevant to Saudi's healthcare sector national objectives}
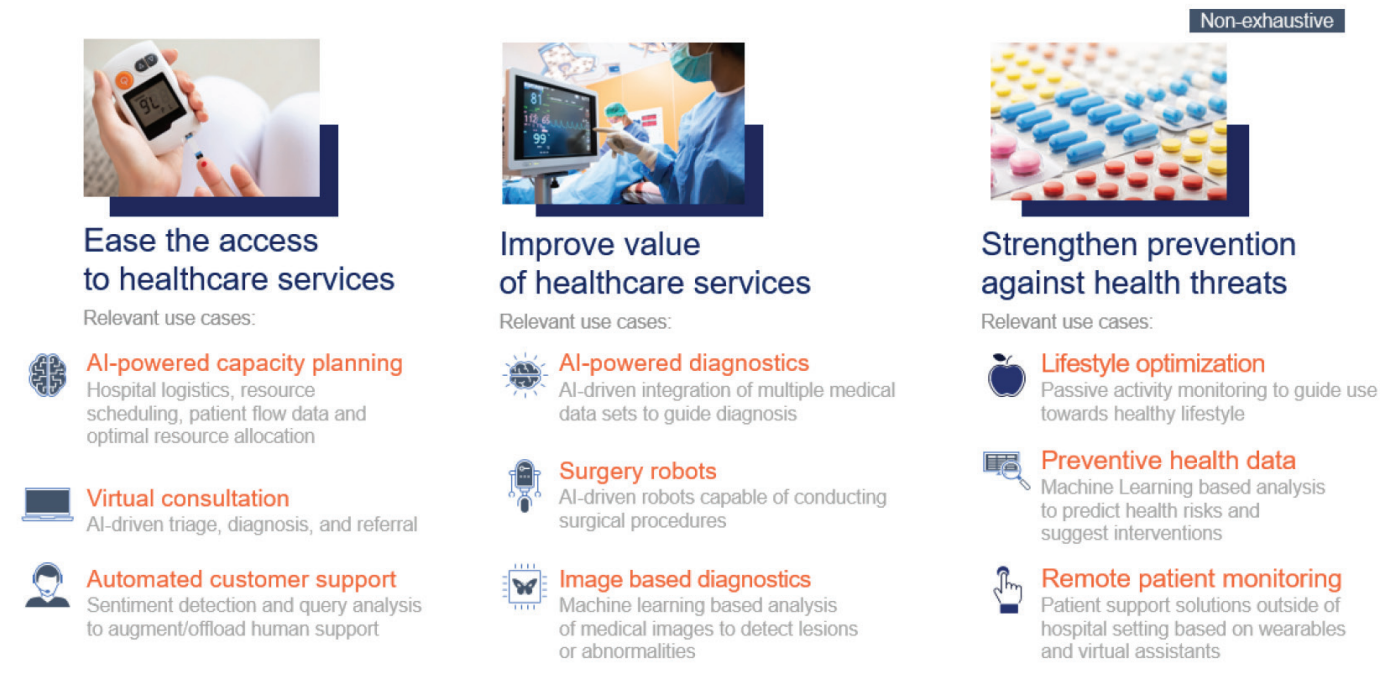

Figure 3 Healthcare strategic objectives with data and AI applications.

healthcare. SDAIA, in line with Vision 2030, is targeting three strategic objectives in the health sector, where it will be working in collaboration with the entire ecosystem to achieve them and advance healthcare as a whole. Figure 3 portrays the three strategic objectives and includes examples of how data and AI could be used in them.

To achieve its ambitious vision, SDAIA has established and activated NCAI, where NCAI's mission is orchestrating AI research and development, providing AI strategic advisory to the government, and promoting AI education and awareness as a sustainable world-class center, leveraging collaborations with academia, public, and private sectors. NCAI is envisaged to raise national AI maturity by co-creating a portfolio of AI use cases along priority sectors (e.g. health) with relevant stakeholders, driving AI use cases development in collaboration with AI partners, and supporting AI research and publications in order to generate impact and achieve sustainable economic growth. NCAI aims to gather and use a range of data types to advance healthcare in the Kingdom, recognizing that the future of healthcare will depend not only on clinical data, but also on behavioral, lifestyle, and genomics data. Saudi Arabia is embarking on a new technological shift by developing both a Biotechnology and a Bioinformatics strategy, which will all work in tandem with the NSDAI to advance healthcare and other industries.

Because Saudi Arabia recognizes the significance and numerous benefits of data and AI technology, it has established a separate entity tasked with leading and advancing this technology on a national scale. SDAIA has already taken several steps toward their goal, as evidenced by how the Kingdom handled the COVID-19 pandemic using digital technologies and how they hosted and organized the Global AI Summit. In regards to the health sector, SDAIA/NCAI has held several workshops to align on priority research and development areas, and is launching research agreements as well as several health programs of excellence.

\section{CONFLICTS OF INTEREST}

The authors declare they have no conflicts of interest.

\section{AUTHORS' CONTRIBUTION}

All authors contributed equally to the writing and review of the final manuscript.

\section{REFERENCES}

[1] Fridsma DB. Health informatics: a required skill for 21st century clinicians. BMJ 2018;362;k3043.

[2] O'Malley AS. Tapping the unmet potential of health information technology. N Engl J Med 2011;364;1090-1.

[3] Rudin RS, Bates DW, MacRae C. Accelerating innovation in health IT. N Engl J Med 2016;375;815-17.

[4] Al-Hanawi MK, Khan SA, Al-Borie HM. Healthcare human resource development in Saudi Arabia: emerging challenges and opportunities - a critical review. Public Health Rev 2019;40;1.

[5] Statistical Yearbook - moh.gov.sa. (n.d.). Available from: https://www.moh.gov.sa/Ministry/Statistics/book/Documents/ book-Statistics-2018.pdf (Retrieved 17 March 2021).

[6] SAMA Working Paper. (n.d.). Available from: http://sama.gov. sa/en-US/EconomicResearch/WorkingPapers/population $\% 20$ aging\%20in\%20saudi\%20arabia.pdf (Retrieved 17 March 2021). 